\title{
A Data Analysis and Evaluation System on LabVIEW
}

\author{
${ }^{\mathrm{a}}$ Lu Qirong, ${ }^{\mathrm{b}}$ Huang Yuanyuan, ${ }^{\mathrm{c}}$ Wang Tao ${ }^{\mathrm{d}}$ Sun Lin
}

\author{
${ }^{a}$ The center of modern education technology Guilin University of Technology Guilin, China \\ ${ }^{b, c}{ }^{d}$ College of Mechanical and Control Engineering Guilin University of Technology Guilin, China
}

\begin{abstract}
The concentration or composition of industrial waste gas is an important parameter in process industry. According to the required of an industrial waste gas monitor and control project a system of data analysis and display is designed based on LabVIEW. The system has 6 modules including error analysis, equal precision data processing, Non-precision data processing, uncertainty evaluation, least squares and regression analysis. Running results indicated that this system is steady and rapidly. It can be used to deal the concentration or composition data accurately in real-time and can be display the result by graph or character. Remarkably the system is designed by LabVIEW, it is easy to modify, upgrade and to use as a subVI in other LabVIEW control system.
\end{abstract}

Index Terms: Data analysis;uncertainty;LabVIEW;waste gas

(C) 2012 Published by MECS Publisher. Selection and/or peer review under responsibility of the International Conference on E-Business System and Education Technology

\section{Introduction}

Since the low ppm-level of $\mathrm{HCL}, \mathrm{NH}_{3}, \mathrm{HF}$ and $\mathrm{CO}_{2}$ tested in the waste gas in the processing industry, and the effect of different density of background gas (like moisture) during the testing process, the test results usually have some error, then the results and truth-values are different which brings the follow-up work great difficulties, the accuracy and sensitivity of the test are also affected. So it is very necessary to have an error analysis to the test results. According to the required of an industrial waste gas monitor and control project a system of data analysis and display is designed based on LabVIEW.

LabVIEW is a graphic programming language. As a kind of graphical programming language, comparing with other programming languages, LabVIEW provides more visualized figures rather than words, while helps faster, more flexible and effective programming as well [1]. In the waste gas analysis system of process industry, by use of the operation and display functions of computers, the data can be collected, processed, displayed and controlled with virtual instruments technology. LabVIEW helps to design the analysis and evaluation platform which has many advantages like easier operation, better handling capacity, flexible expansion and etc. Running results indicated that this system is steady and rapidly. It can be used to deal the concentration or composition data accurately in real-time and can be display the result by graph or character. It also could satisfy the need of

Corresponding author:

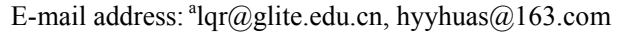


online waste gas testing on the aspects such as the data processing speed, accuracy and capacity. Finally, the articles combined of the gas concentration which collected by the gas detection sensor as an example in order to introduce the error analysis and the function of the evaluation of common platforms, also to verify the validity of the platform.

\section{Error Analysis Theory}

In accordance with the nature and basic are features of errors. There are many different types of errors that can occur in a measurement, but they will generally be classified as three categories [2] [3]: random errors, systematic errors and gross errors.

\subsection{Random errors}

In the measurement, though the systematic errors have been gotten rid of successfully, the result of the repeated measurements of the same physical quantity under same conditions are of a little difference. Thus the absolute value and the sign of errors produced are always changing unexpectedly. This phenomenon is called random errors [2]. According to statistical methods, we used overall average size and dispersion to obtain an estimate of the random errors [4].

The formula for calculating the arithmetic mean:

$\bar{x}=\frac{1}{n} \sum_{i=1}^{n} x_{i}$

The estimated value of standard deviation by Bessel equation:

$\sigma=\sqrt{\frac{1}{n-1} \sum_{i=1}^{n} v_{i}^{2}}$

\subsection{Systematic errors}

Systematic error is the error that is constant or based on a rule change in a series of repetitions of the same measure condition [2]. Usually, systematic error is defined as the expected value of the overall error.

Criterion of system error [4] [6]:

\section{1) Abbe-Helmert criterion}

According to Measurement sequence residual will order, if meet the conditions:

$\left|v_{1} v_{2}+v_{2} v_{3}+\cdots+v_{n-1} v_{n}\right|=\left|\sum_{i=1}^{n-1} v_{i} v_{i+1}\right|>\sqrt{n-1} \sigma^{2}$

Then may be existed periodicity system error.

\section{2) Malcolm criterion}

Around the residual $\mathrm{v}_{\mathrm{i}}$ according to the arranged in order, then divides into two halves and sums.

When $\mathrm{n}$ is even:

$D=\sum_{i=1}^{n / 2} v_{i}-\sum_{i=n / 2+1}^{n} v_{i}$

When $\mathrm{n}$ is odd: 
$D=\sum_{i=1}^{(n+1) / 2} v_{i}-\sum_{i=(n+1) / 2+1}^{n} v_{i}$

If $\mathrm{D} \approx 0$, then there is no progressive error in measurement data; Otherwise, the progressive error is exist.

\section{3) Residual sum of the Criterion}

If the residual $\mathrm{v}_{\mathrm{i}}$ meet the conditions:

$$
\left|\sum_{i=1}^{n} v_{i}\right|>2 \sigma \sqrt{n}
$$

Then may be existed periodicity system error.

\section{4) Standard deviation of comparison}

Using different methods to calculate the standard deviation, then through the comparison discovered whether to existence the system errors.

Bessel equation:

$\sigma_{1}=\sqrt{\frac{1}{n-1} \sum_{i=1}^{n} v_{i}^{2}}$

Peter's equation:

$\sigma_{2}=1.253 \frac{\sum_{i=1}^{n}\left|v_{i}\right|}{\sqrt{n(n-1)}}$

If

$\frac{\sigma_{2}}{\sigma_{1}} \geq 1+\frac{2}{\sqrt{n-1}}$

The system errors may be existed.

\subsection{Gross errors}

Gross error is the error that caused the measured values deviate significantly from the actual value in the measurement of certain conditions [3]. This value is measured as bad and should be removed .Only after the elimination of gross errors, the measurement data can be processing.

Criterion of gross errors [4] [6]:

1) Letts' criterion

When measurement data obey normal distribution and have sufficient number of measurements, if the absolute value of the residuals to meet the conditions:

$\left|v_{i}\right|=\left|x_{i}-\bar{x}\right|>3 \sigma$ 
The measurement data may be error data and should be eliminated.

2) Chauvenet criterion

If repeated measured a physical parameter $\mathrm{n}$ times in the same measure condition. The measurement data are $\mathrm{x}_{1}, \mathrm{x}_{2} \ldots \mathrm{x}_{\mathrm{n}}$. If the absolute value of the residuals to meet the conditions:

$\left|v_{j}\right|=\left|x_{j}-\bar{x}\right|>Z_{c} \sigma$

Zc: Chauvenet factors.

The measurement data may be error data and should be eliminated. As $\mathrm{Zc}<3$, The Chauvenet criteria will be able to fill in the vacancies of Letts' criterion with a certain extent.

\section{3) Lomnaofski norm}

If the absolute value of the residuals to meet the conditions:

$\left|v_{j}\right|=\left|x_{j}-\bar{x}\right|>K(n, a) \sigma$

$\mathrm{K}(\mathrm{n}, \mathrm{a})$ : $\mathrm{t}$ modified coefficient

$\mathrm{n}$ : measuring times; a: Saliency

The measurement data may be error data and should be eliminated. Its characteristics are first removed a suspicious measurement data, and then according to the $t$ distribution to test whether the excluded measurement data is outliers.

\section{Process Industrial Emissions on-line data processing and analysis}

Error Analysis and Evaluation of the common platforms, which include the equal precision data processing, Non-precision data processing, uncertainty evaluation, error analysis, effective data processing, least squares and regression analysis module. The data analysis system interface shown in Fig.1.

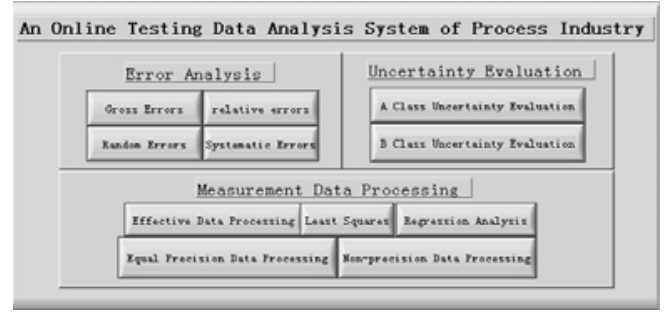

Figure 1. An Online Testing Data Analysis System of Process Industry

The following combination of the gas concentration which collected by the gas detection sensor as an example in order to introduce the error analysis and the function of the evaluation of common platforms, also to verify the validity of the platform.

\section{1 Error analysis and data processing}

The Error analysis is including the systematic error analysis module, gross error analysis module, random error analysis module, as well as relative error analysis module.

The systematic errors analysis module interface shown in Fig.2. According to Abbe-Helmert criterion, the led is not lit, the measurement data in an error-free; and if the led is lit, the measurement data in an error. At this time we should find out the reasons and correct or eliminate the system error then re-measured. 


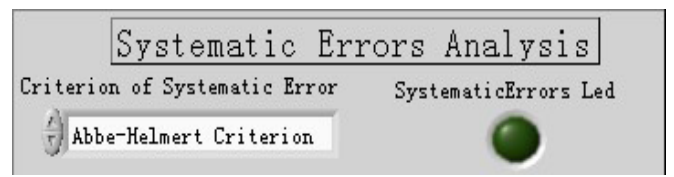

Figure 2. systematic error analysis module

The gross error analysis module interface shown in Fig.3.According to Wright criteria criterion, we obtain the ordinal data 3 , data value is 21.85 . It is a bad value and should be struck out. After removing the bad value, then re-processing the residual measurements, we can obtain the Arithmetic mean and standard deviation estimated value.

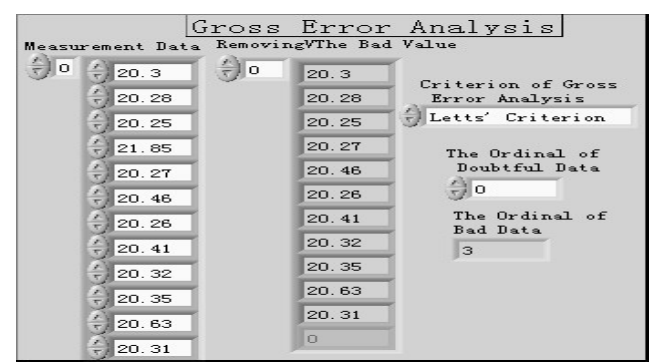

Figure 3. gross error analysis module

The random error analysis module interface shown in Fig.4. We usually use the arithmetic mean of multiple measurements of Random error analysis as the final result; the estimated value of standard deviation shows the probability distribution of random errors. If standard deviation is large, the distribution range is wide and the precision is low; On the contrary, if standard deviation is small, it means small distribution and high precision. From the interface, probability density chart shows that random error distribution of the measurement is narrow and high precision.

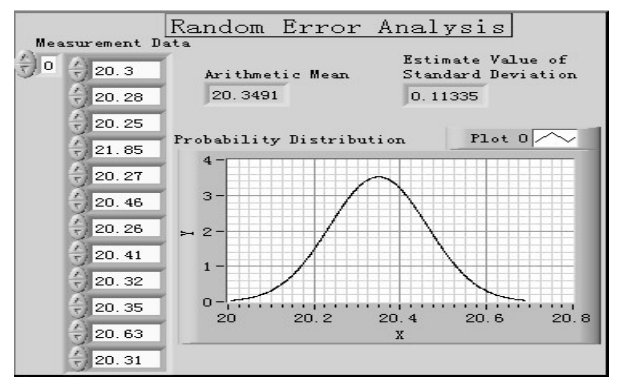

Figure 4. random error analysis module

The relative error analysis module interface shown in Fig.5.In the module get both the absolute and the relative error of the measurement, the error shows the degree and accuracy that the measured value deviating from the actual.

The interface of uncertainty evaluation in platform is shown in Fig.6. The final result is not an objective value, and it must not be. It is only an approximation of the true value. Therefore, we must make more accuracy estimates of the estimate value. The uncertainty module in Fig. 6 is used to reflect and assess the uncertainty of the measured value as error existing in measurement. Uncertainty [7] is an important indicator of the quality level of evaluation measurement. 


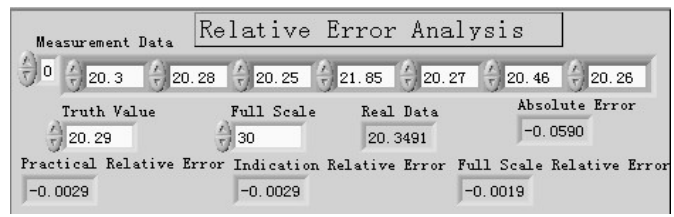

Figure 5. relative error analysis module

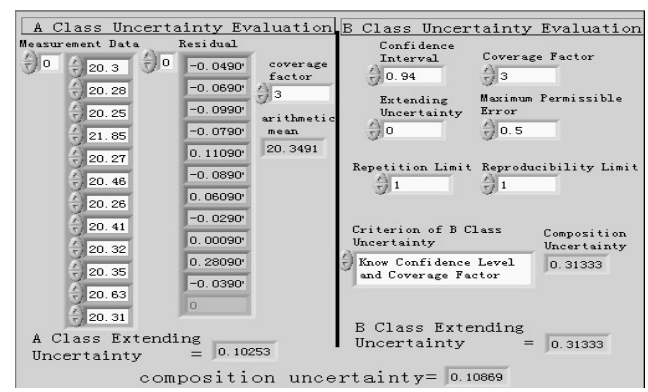

Figure 6. Uncertainty Module

Module designs A class and B class methods for uncertainty evaluation. Class A uses statistical analysis ,as the factor included in the module is related to the freedom and confidence level of measured values, Class B has not use statistical analysis, but on the other methods to estimate the probability distribution or distribution assumption to evaluate the standard deviation, and it includes five evaluation methods ,such as the known confidence level and coverage factor, the known expanded uncertainty and coverage factor, the known grade of the instrument, the known repetition and reproducibility limit, in the five different ways, you are free to select the desired method of the platform according to the actual needs.

Platform also design an effective digital processing module, bits of the effective number of digits expressed a certain measurement accuracy, which means you can write numbers no more and no less. The Rounding rules takes "When the dealled number is 6 the last number is add 1, When the dealled number is 5 according the parity of the last number." principle to process data. That the interface of effective digital processing shown in Fig.7.

\begin{tabular}{|c|c|c|c|}
\hline $\begin{array}{l}\text { Original Date } 1 \\
\text { Jy } 10.345\end{array}$ & $\begin{array}{l}\text { Original Date } 2 \\
\frac{10.355}{5}\end{array}$ & $\begin{array}{l}\text { Original Date } 3 \\
\frac{\sqrt[A]{7}}{10.354}\end{array}$ & $\begin{array}{l}\text { Original Date } 4 \\
\text { (s) } 10.356\end{array}$ \\
\hline Rounded Data 1 & Rounded Data 2 & Rounded Data 3 & Rounded Data 4 \\
\hline 10.34 & 10.36 & 10.35 & 10.36 \\
\hline
\end{tabular}

Figure 7. Effective digital processing module

Now, we take error analysis of the $\mathrm{CO}_{2}$ gas concentration data on above, so as uncertainty evaluation and effective digital processing, the final analysis interface of the equal precision data processing shown in Fig. 8 . 


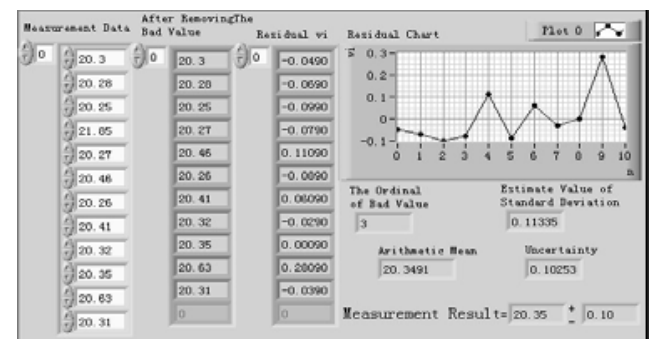

Figure 8. Equal precision of data processing module

By Fig.8, we can see, the residual $v_{i}$ largely looks the same in positive and negative, no significant changes in law, according to Residual error observation criterion, we can further describe that no significant progressive or periodic system error of the measurement data. The final result is measured: $20.35 \pm 0.10$.

In the above example, if in different conditions (using different measurement methods, different times of measurement or different surveyor), we measured the gas concentration twice, And gain measurement values 1 and 2.At this time, with Non-precision approach, we take error analysis and data processing. By the same token, through error analysis, uncertainty evaluation, and effective digital processing, the interface of non-precision data is processing shown in Fig.9. The "coverage factor" in the interface is determined by the confidence coefficient.

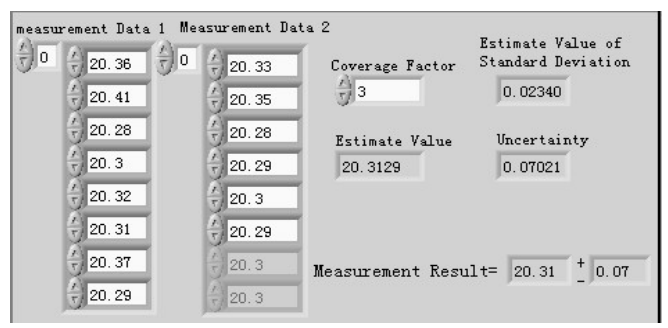

Figure 9. Unequal accuracy data processing module

Enter the measurement data in the platform, set the "coverage factor" as"3." After running the program, the final measurement result is $20.31 \pm 0.07$.

\section{2 Using the least square method and Regression Analysis to process data}

In the error theory, we commonly use least square method and regression analysis to define the empirical formula. It is also applied in this platform to obtain the formula between the two variables.

Take the $\mathrm{CO}_{2}$ gas detection sensors for example, the measurement values of gas concentrations and voltage shown in figure 10, then, set up the operating parameters, input measurement data and run the program, you will get the results in Fig.10.

Comparing Fig.10 and Fig.11, Shows that, the two methods in dealing with the measured data of linear relationship are consistent. It also demonstrates the effectiveness of the platform. If you deal with the non-linear relationship, you can convert to the form of linear relationship by variable substitution, and then use the least square method or regression analysis to find the unknown parameters, and determine the empirical formula. 


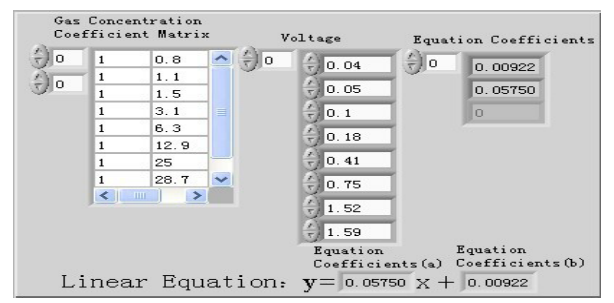

Figure 10. Least Squares

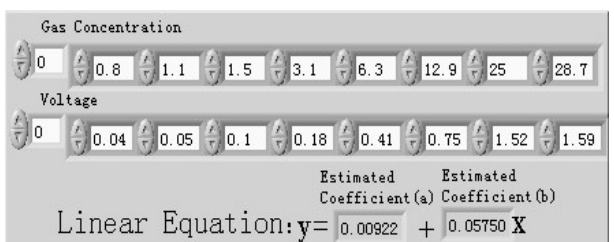

Figure 11. Linear regression

\section{Conclusion}

A visual virtual instrument testing interface could be built through the LabVIEW, and the tested data's accuracy are improved after processed by the designed error analysis and evaluation common platform, the precision and sensitivity of the waste gas's online testing and analysis system are increased as well. Remarkably the system is designed by LabVIEW, it is easy to modify, upgrade and to use as a subVI in other LabVIEW control system. Then the testing results of waste gas with low ppm-level like $\mathrm{HCL}, \mathrm{NH}_{3}, \mathrm{HF}$ and $\mathrm{CO}_{2}$ could be more precise and reliable. At the same time, the platform adopts a fairly effective and sound way of error analysis and data processing, then the process is faster and more precise, and the tested data are more reliable and practical.

\section{Acknowledgements}

This research was supported by Guangxi Science Foundation (0991253). The staffs of the center of modern education technology also gave help for us. Thanks for all people.

\section{REFERENCES}

[1] Xuedong Jin,Evaluation of measurement uncertainties of virtual instruments, Sringer-Verlag London,30 March 2005.

[2] JAYNES E. T, "Information theory and statistical mechanics," PhysicalReview , vol. 106 (4), pp. 620-630,1957.

[3] Warnick, K. F., Weng Cho Chew, "Error analysis of the moment method," Antennas and Propagation Magazine, IEEE, Vol.46(6), pp.38-53, Dec 2004.

[4] R.B.Streets, "Arbitrary Non-Mean-Square Error Criteria," IEEE Transaction on Automatic Control, pp.376-377, October 1963.

[5] M. Zakai, "General Error Criteria," IEEE Transactions on Information Theory, pp. 94-95, January 1964.

[6] Xing Li, Weiru Fang, Qi Tian , "Error criteria analysis and robust data fusion," IEEE International Conference, vol.4, pp.37-40, Apr 1994.

[7] Furness.R, "The cost of measurement uncertainty," PCIC Europe, pp.1-8 ,June 2008 . 